\title{
NATURAL KILLER CELLS AND IMMUNOTHERAPY BASED ON MONOCLONAL ANTIBODIES
}

\author{
Magdalena Pencheva-Demireva $^{1^{*} \text {, Katerina Kavaldzhieva }}{ }^{\mathbf{1}}$, Nikola Mladenov ${ }^{1}$, Vladislav Lazarov ${ }^{1}$, \\ Tzvetanka Markova ${ }^{2}$, and Dimitrina Dimitrova-Dikanarova ${ }^{1}$ \\ ${ }^{1}$ Department of Biology, Medical University, Sofia, Bulgaria, ${ }^{2}$ Department of Pharmacology and \\ Toxicology, Medical University, Sofia, Bulgaria
}

Natural killer (NK) cells are effector lymphocytes of innate immunity needed to protect against stressed cells and to destroy tumor cells and virus-infected cells. These cells play an important role in the immune surveillance of malignant cells, preventing their uncontrolled growth. Natural killer cells recognize target cells directly through their receptors, which bind to various determinants on the surface of the target cell. The receptor-ligand (secretory or membrane-bound) interaction between the NK cells and the target cells determines NK's cell activity. The use of monoclonal antibodies in tumor therapy has increased significantly in the recent years. These antibodies are intended to block inhibitory receptors (immune checkpoint inhibitors) expressed by immune cells or to block their ligands expressed by tumor cells. Examples of such immune checkpoint molecules are the following receptors: cytotoxic T lymphocyte-associated protein 4 (CTLA-4), programmed cell death protein 1 (PD-1) and others. This blocking inhibits tumor growth by enabling immune system reactivation. The advances in cellular immunobiology that have provided the establishment of blocking monoclonal antibodies (ipilimumab, nivolumab, etc.) and increased NK cell proliferative activity are promising therapies for neoplasms. Biomed Rev 2020; 31: 61-65

Key words: lymphocytes, inhibitory receptors, cancer, PD-1, CTLA-4

\section{INTRODUCTION}

Tumor cells use various mechanisms to prevent their recognition and subsequent attack from the immune system. In the macroorganism tumor cell destruction is carried out mainly by cytotoxic T cells and natural killer cells. Natural killer (NK) cells destroy target cells through several basic mechanisms, two of which require direct contact between the target cell and the NK cell. In the first mechanism, the activated natural killer cell performs its cytolytic action by degranulation of perforins and granzymes contained in the

*Correspondence to: Dr Magdalena Pencheva-Demireva, Department of Biology, Medical University,

2 Zdrave Street, BG-1431 Sofia, Bulgaria

Tel: +359 29172 648; E-mail: mpdemireva@medfac.mu-sofia.bg 
granules, which destroy the target cell. This mechanism is very effective and is realized by activating apoptotic cysteine proteases, called caspases, characteristic of the early stages of apoptosis. The second mechanism involves the participation of apoptotic receptors (e.g., first apoptosis signal receptor, Fas/CD95) in the membrane of the target cell and their ligands (e.g., first apoptosis signal ligand, FasL/CD95L) in the membrane of a NK cell, which leads to the classical caspase-dependent apoptosis (1). In both mechanisms, the effector function of NK cells is regulated by receptor-ligand interactions, as the NK cell could be carrying either the receptor or the ligand (2), and could therefore be regulated at this level. Natural killer cells also express the FcyRIIIA and/or Fc $\gamma$ RIIC receptor (s), which bind to the Fc fragment of an immunoglobulin molecule. When an antigenic epitope of the target cell is bound to the paratope of an immunoglobulin molecule, the NK cell binds to its Fc region and lyses the target cell without priming. The NK cell activated by this mechanism secretes cytokines and interferon gamma (IFN- $\gamma$ ), which enhance the immune response. The antibodydependent cellular cytotoxicity (ADCC) of NK cells against cancer cells is used in the treatment of neuroblastoma, breast cancer, B-cell lymphoma and other neoplasms that express large amounts of tumor-specific antigens (TSAs).

Cells that are under stress can be recognized by NK cells and activate their cytotoxic properties. The Natural Killer Group 2D (NKG2D) receptor, which is expressed by NK cells, recognizes and binds to cell stress ligands, such as polypeptide sequences $\mathrm{A}$ and $\mathrm{B}$, which are combined with major histocompatibility complex class I (MHC class I) molecules (MHC class I polypeptide-related sequence $\mathrm{A}$ and B, MICA / MICB). These ligands are expressed under cellular stress of various etiologies: such as viral infection, malignant transformation, and DNA damage (3-5).

Another mechanism by which NK cells can recognize an infected or transformed cell is through the „missing self“ (6, $7)$. When a nuclear cell: does not express molecules of MHC class I; expresses them to a very low degree or there is a change in the structure of these molecules, this signals the natural killers to lyse the corresponding cell (8). Natural killer cells also express receptors called immunoglobulin-like receptors (Killer-cell immunoglobulin-like receptor, KIR). The KIR receptors of NK cells interact with MHC class I molecules of the target cells. This allows them to detect virus-infected cells or cancer cells.

Tumor immunoediting is described as a dynamic interaction between carcinoma and the host immune system, manifested in three phases: elimination, balance, and avoidance (9). In the elimination phase, the immune system can identify and eliminate tumor cells that express tumor-associated antigens (TAAs). NK cells, which are „naturally“ equipped with all the necessary attributes to recognize and remove tumor cells, are activated in the earliest stages of tumorigenesis. The balance phase is characterized by tumor latency, i.e. tumor cells are not destroyed, but their growth is immune-restricted. Avoidance of immune system surveillance is achieved by accumulation of mutations, expression of receptors close in nature to healthy cell receptors, expression of inhibitory ligands / receptors, or lack of receptors $(6,9)$.

\section{IMIMUNE CHECKPOINTS}

Tumor cells often carry proteins that reveal the oncological nature of the cells. Sometimes, however, they commit "identity theft" from a non-transformed cell by expressing healthy cell proteins. Recent studies (10-12) show that cancer cells use immune checkpoints to suppress and ,escape' the attack of the immune system. T cells and NK cells do not attack tumor cells and they remain undisturbed. Many types of oncological diseases "escape" from the immune system in this way, by inhibiting the T cell signal and the NK cell signal. Cytotoxic T lymphocyte-associated protein 4 and PD-1 are two molecules on the $\mathrm{T}$ and $\mathrm{NK}$ cell membrane identified as key inhibitors of $\mathrm{T}$ and NK cell function. Close in function to cytotoxic $\mathrm{T}$ lymphocytes, NK cells also express inhibitory receptors with which tumor cells interact in order to "hide“ from attacks. Such inhibitory checkpoints expressed on NK cells are CTLA-4, PD-1, LAG-3 (Lymphocyte-activation gene 3), TIM-3 (T-cell immunoglobulin and mucin domain-3) (Fig. 1) (13).

The treatment of malignant diseases by injecting antitumor antibodies derived from different animal species has long been experimented with, but has always been followed by severe toxic conditions (14). Only after the introduction of hybridoma technology for production of monoclonal antibodies it became possible to amount quantity needed for an entire treatment course. Thus, experimental passive immunotherapy was introduced as a reliable therapeutic method that could be applied in clinics.

A major problem with monoclonal antibody immunotherapy has been the response of the patient's immune system to injected foreign immunoglobulins. This can be done by substituting a constant region by the CDR (Complementary Determing Regions) grafting technique from a murine antibody 


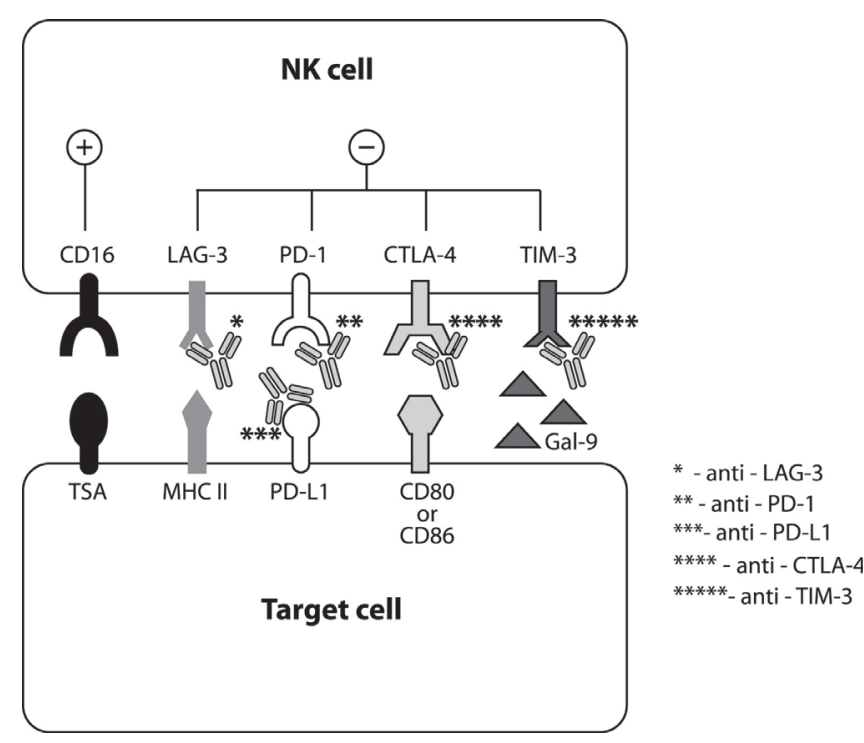

Figure. 1. Inhibitory checkpoints (receptors) expressed by the NK cells. The interaction between the inhibitory receptor (LAG-3, PD-1, CTLA-4, TIM-3) expressed by the NK cells and their ligands on the target cells (MHC II, PD-L1, CD80 / CD86, Galectin-9 in its secreted form), can be blocked by monoclonal antibodies (mAbs) (examples are given with an asterisk *). These blocking antibodies can be directed against: the receptor; the ligand; as well as against the receptor and ligand. Using this mechanism of preventing receptor-ligand contact, the NK cells can increase their cytolytic properties and attack the target cell. The activating CD16 receptor expressed by the NK cell, binds to the tumor-specific antigen (TSA), expressed by the target cells. When this connection is blocked by $m A b$, the NK cells are activated and attack the target cells. From (13), modified.

with a human immunoglobulin constant region. Studies show that humanized antibodies reduce their immunogenicity to the human body (14).

\section{NK CELLS, MALIGNANCIES AND THEIR IMMUNOTHERAPY}

Treatment of malignant tumors with monoclonal antibodies is based on the following mechanisms: (i) activation of effector cells that produce antitumor effector molecules from the immune system, (ii) after binding of the antibody to the tumor cell, there was an impact on the processes of growth and differentiation, (iii) induction of active immunization with monoclonal antibody by the mechanism of the anti-idiotypic vaccine, (iv) indirect activation of other biologically active agents such as IFN- $\gamma$, IL-2 and others, and (v) monoclonal antibody blocks important mechanisms for cell proliferation of tumor cells (14).

There are various antibody-based tumor immunotherapies. An example of such therapy is - antibody bound to a drug or toxin by „spacer“ molecules (most commonly human albumin or various dextrans). It is assumed that the antibody-bound preparation will be delivered by the antibody to the target cell. An alternative method of immunotherapy that is widely used is treatment with monoclonal antibodies associated with plant or bacterial toxins. Many of the toxins used are composed of two protein chains: the B-chain, which binds to the target cell, and the A-chain, which inhibits cell protein synthesis. A serious drawback in the use of toxins is their immunogenicity. Monoclonal antibodies can be associated with radionuclides. Radiolabeled monoclonal antibodies can „kill“ cells that are at a considerable distance from the target cell to which the isotope-labeled antibody has bound (14).

Antibodies remain a relatively reliable tool in complex therapy against malignanciesIt should not be forgotten that the diversity in the forms of malignant tumors necessitates the search of a specific therapy for each specific form. Each tumor and each stage of its development requires a specific therapeutic agent or a combination of several. Patients undergoing immunotherapy must also be well selected in order to achieve the wanted effect.

The use of monoclonal antibodies to block immune checkpoints is the latest and most widely studied option for immunotherapy. In 2018 James Allison and Tasuku Honjo were awarded the Nobel Prize in Physiology or Medicine for their discoveries in the field of tumor immunotherapy "to detect cancer treatment by inhibiting negative immune regulation". Shortly after the first publications related to the new type of immunotherapy using blocking antibodies such as anti-CTLA-4 and anti-PD-1, one of us (TM) together Japanese colleagues from Hyogo College of Medicine, Nishinomiya published an article reporting an improvement of the already discovered therapy (15).

The immune checkpoint inhibitor is a monoclonal antibody that, triggers an attack by the immune system against tumor cells (10). Therapy with these drugs has led to impressive success in recent years, especially in patients with metastatic melanoma or Hodgkin's lymphoma (16) and appears promising for patients with other types of neoplasms.

Inhibitors of immune checkpoints block proteins on cancer cells or on the surface of $\mathrm{T}$ cells, NK cells that respond to 
these points. The goal of receptor inhibition is to eliminate the signals that interfere with the functions of cytotoxic $\mathrm{T}$ cells and NK cells, so these cells can recognize cancer cells and attack them.

Three immune checkpoint inhibitors have received rapid approval from the US Food and Drug Administration (FDA) against cancer, including ipilimumab (Yervoy $®$ ), pembrolizumab (Keytruda $\left.{ }^{\circledR}\right)$ and nivolumab (Opdivo $\left.{ }^{\circledR}\right)$. This type of immune control therapies are one of the most promising treatments against malignant tumors.

Immunotherapies targeting the CTLA-4 checkpoint open up new possibilities in the field of human oncology. Two antagonistic monoclonal antibodies against CTLA-4 ipilimumab (IgG1 isotype) and tremelimumab (IgG2 isotype), block the interaction between CTLA-4 and its co-stimulatory ligands - CD80 and CD86 (ligands of the activating checkpoint CD28). These drugs in the form of inhibitors are approved for the treatment of advanced cancer. The exact mechanisms by which anti-CTLA-4 works are not fully understood. Data to date suggest that anti-CTLA-4 acts mainly in secondary lymphoid organs by releasing effector $\mathrm{T}$ cells from the inhibitory effect, by depleting/reducing the number of Treg cells in the tumor microenvironment $(11,17)$.

Despite promising early results from monoclonal antibody therapy against CTLA-4 in human clinical trials, levels of objective response remain low in most cases. Improvements have been observed when anti-CTLA- 4 is combined with the chemotherapeutic drug dacarbazine (12). However, the best results were observed when combination therapy with antiCTLA-4 monoclonal antibody and blocking antibody against PD-1 was administered $(18,19)$.

Anti-PD-1 is another monoclonal antibody that successfully blocks the receptor-ligand bond between the effector and tumor cells. It is used on its own to treat cancer in humans, as well as in combination with another antibodies or medicine. Combination therapies with anti-CTLA-4 and anti-PD-1 have achieved high efficacy in patients with stage III and IV melanoma $(19,20)$. Immunotherapy with anti-PD-1 focuses largely on stimulating T-cell antitumor responses by blocking the binding of PD-1 to its ligands PDL1 (B7-H1, CD274) and PD-L2 (B7-DC, CD273). Blocking $\mathrm{PD}-1$ removes the inhibitory effects that occur as a result of the PD-1-PD-L1 interaction (21-23). PD-L1 and PD-L2 proteins are largely not expressed by healthy cells. PD-L1 expression is increased in more than 20 types of human tumors (24). In addition, cancer cells increase the expression of PD-L1 in response to inflammatory cytokines such as IFN- $\gamma$, as well as non-malignant cells found in the tumor microenvironment (25). These experimental data indicate that IFN- $\gamma$ would improve therapy with anti-PD-1 and / or anti-PD-L1 / L-2 monoclonal antibody, as it increases the expression of these ligands and blocking the receptor-ligand bond will be successful.

The era of anti-tumor checkpoint blockade includes the synthesis and clinical testing of anti-PD-1 monoclonal antibodies - nivolumab and pembrolizumab. Another way to target the signaling pathway is through antibodies that bind the PD-L1 ligand, which is expressed by tumor cells. PD-L1 targeting is thought to be less toxic because it acts by selectively affecting the immune response in the tumor microenvironment and is not affected by the degree of PD-L1 expression (24).

\section{CONCLUSION}

Despite the great progress that has been made in the treatment of oncological diseases, there are still limitations such as unclear mechanisms, side effects, poor effectiveness of treatment and others. This requires the research of new new approaches (personalized medicine) and drugs, finding more effective carriers of these drugs, which would allow selective entry into the cell, and hence reduce the side effects of the drug, as well as the use of combination therapy.

\section{ACKNOWLEDGMENTS}

This work was supported by the National Research Program "Young Scientists and Postdoctoral Candidates" and by Memorandum between Medical University, Sofia, Bulgaria and Hyogo College of Medicine, Nishinomiya, Japan from 24th of April 2013. We thank Dr Carotta for kindly letting us to use and modify his figure, the Figure 1 of the present review.

\section{CONFLICT OF INTEREST}

None.

\section{REFERENCES:}

1. Mitra R, Singh S, Khar A. Antitumor immune responses. Exp Rev Mol Med 2003; 5(3):1-19. DOI: 10.1017/ S1462399403005623.

2. Smyth MJ, Cretney E, Kelly JM, Westwood JA, Street $\mathrm{SE}$, Yagita $\mathrm{H}$, et al. Activation of NK cell cytotoxicity. Mol Immunol 2005; 42(4):501-510. DOI: 10.1016/j. molimm.2004.07.034 
3. Groh V, Rhinehart R, Secrist H, Bauer S, Grabstein $\mathrm{KH}$, Spies T. Broad tumor-associated expression and recognition by tumor-derived gamma delta $\mathrm{T}$ cells of MICA and MICB. Proc Natl Acad Sci USA 1999; 96(12):6879-6884. DOI: 10.1073/pnas.96.12.6879

4. Groh V, Rhinehart R, Randolph-Habecker J, Topp MS, Riddell SR, Spies T. Costimulation of CD8 alpha beta $\mathrm{T}$ cells by NKG2D via engagement by MIC induced on virus-infected cells. Nat Immunol 2001; 2(3):255-260. DOI: $10.1038 / 85321$

5. Jinushi M, Takehara T, Tatsumi T, Kanto T, Groh V, Spies $\mathrm{T}$, et al. Expression and role of MICA and MICB in human hepatocellular carcinomas and their regulation by retinoic acid. Int J Cancer 2003; 104(3):354-361. DOI: 10.1002/ ijc. 10966

6. Ljunggren HG, Kärre K. In search of the ,missing self": MHC molecules and NK cell recognition. Immunol Today 1990; 11(7):237-244. DOI: 10.1016/01675699(90)90097-s

7. Watzl C. The NKG2D receptor and its ligands-recognition beyond the ,missing self"? Microbes Infect 2003; 5(1):3137. DOI: $10.1016 / \mathrm{s} 1286-4579(02) 00057-6$

8. Morvan MG, Lanier LL. NK cells and cancer: you can teach innate cells new tricks. Nat Rev Cancer 2016; 16(1):7-19. DOI: $10.1038 / \mathrm{nrc} .2015 .5$

9. Schreiber RD, Old LJ, Smyth MJ. Cancer immunoediting: integrating immunity's roles in cancer suppression and promotion. Science 2011; 331(6024):1565-1570. DOI: 10.1126/science. 1203486

10. Pardoll DM. The blockade of immune checkpoints in cancer immunotherapy. Nat Rev Cancer 2012; 12(4):252264. DOI: $10.1038 / \mathrm{nrc} 3239$

11. Kvistborg P, Philips D, Kelderman S, Hageman L, Ottensmeier C., Joseph-Pietras, D, et al. Anti-CTLA-4 therapy broadens the melanoma-reactive CD8+ T cell response. Sci Transl Med 2014; 6(254):254ra128. DOI: 10.1126/scitranslmed.3008918

12. Ribas A, Kefford R, Marshall MA, Punt C, Haanen $\mathrm{JB}$, Marmol M, et al. Phase III randomized clinical trial comparing tremelimumab with standard-of-care chemotherapy in patients with advanced melanoma. $J$ Clin Oncol 2013; 31(5):616-622. DOI: 10.1200/ JCO.2012.44.6112

13. Carotta S. Targeting NK Cells for Anticancer Immunotherapy: Clinical and Preclinical Approaches. Front Immunol. 2016;7:152. DOI: 10.3389/fimmu.2016.00152

14. Kehayov I, Kyurkchiev S. Compendium of immunology. Venimeks, Sofia, Bulgaria. 1999, 110-117.

15. Ma Z, Li W, Yoshiya S, Xu Y, Hata M, El-Darawish
Y et al. Augmentation of Immune Checkpoint Cancer Immunotherapy with IL18. Clin Cancer Res 2016; 22(12):2969-2980. DOI: 10.1158/1078-0432.CCR-15-1655

16. Syn NL, Teng MWL, Mok TSK, Soo RA. De-novo and acquired resistance to immune checkpoint targeting. Lancet Oncol 2017; 18(12):e731-e741. DOI:10.1016/ S1470-2045(17)30607-1

17. Twyman-Saint VC, Rech AJ, Maity A, Rengan R, Pauken $\mathrm{K}$, Stelekati E et al. Radiation and dual checkpoint blockade activate non-redundant immune mechanisms in cancer. Nature. 2015; 520(7547):373-7. DOI: 10.1038/ nature 14292

18. Wolchok JD, Kluger H, Callahan MK, Postow MA, Rizvi NA, Lesokhin AM et al. Nivolumab plus ipilimumab in advanced melanoma. $N$ Engl J Med. 2013; 369(2):122-33. DOI: 10.1056/NEJMoa1302369

19. Postow MA, Chesney J, Pavlick AC, Robert C, Grossmann $\mathrm{K}, \mathrm{McDermott} \mathrm{D}$ et al. Nivolumab and ipilimumab versus ipilimumab in untreated melanoma. N Engl J Med. 2015; 372(21):2006-17. DOI: 10.1056/NEJMoa1414428

20. Larkin J, Hodi FS, Wolchok JD. Combined Nivolumab and Ipilimumab or Monotherapy in Untreated Melanoma. N Engl J Med. 2015; 373(13):1270-1271. DOI: 10.1056/ NEJMc1509660

21. Chatterjee P, Patsoukis N, Freeman GJ, Boussiotis VA. Distinct roles of PD-1 ITSM and ITIM in regulating interactions with SHP-2, ZAP-70 and Lck, and PD-1mediated inhibitory function. Blood 2013;122:19. DOI: 10.1182/blood.V122.21.191.191

22. Chemnitz JM, Parry RV, Nichols KE, June CH, Riley JL. SHP-1 and SHP-2 associate with immunoreceptor tyrosine-based switch motif of programmed death 1 upon primary human $\mathrm{T}$ cell stimulation, but only receptor ligation prevents T cell activation. J Immunol 2004; 173(2):945-954. DOI: 10.4049/jimmunol.173.2.945

23. Sheppard KA, Fitz LJ, Lee JM, Benander C, George GA, Wooters J, et al. PD-1 inhibits T-cell receptor induced phosphorylation of the ZAP70/CD3zeta signalosome and downstream signaling to PKCtheta. FEBS Lett 2004; 574(1-3):37-41. DOI: 10.1016/j.febslet.2004.07.083

24. Zou W, Chen L. Inhibitory B7-family molecules in the tumour microenvironment. Nat Rev Immunol 2008; 8(6):467-477. DOI: $10.1038 /$ nri2326

25. Karachaliou N, Gonzalez-Cao M, Crespo G, Drozdowskyj A, Aldeguer E, Gimenez-Capitan A, et al. Interferon gamma, an important marker of response to immune checkpoint blockade in non-small cell lung cancer and melanoma patients. Ther Adv Med Oncol 2018; 10:1758834017749748. DOI: $10.1177 / 1758834017749748$ 